EPJ Web of Conferences 59, 08005 (2013)

DOI: $10.1051 /$ epjconf/20135908005

(C) Owned by the authors, published by EDP Sciences, 2013

\title{
Cryogenic cooling for high power laser amplifiers
}

\author{
J.P. Perin ${ }^{1, a}$, F. Millet ${ }^{1}$, M. Divoky ${ }^{2}$ and B. Rus ${ }^{2}$ \\ 1 CEA/INACISBT, 17 Rue des Martyrs, 38054 Grenoble Cedex 9, France \\ 2 Institute of Physics of the ASCR, v.v.i. 18221 Prague 8, Czech Republic
}

\begin{abstract}
Using DPSSL (Diode Pumped Solid State Lasers) as pumping technology, PW-class lasers with enhanced repetition rates are developed. Each of the Yb YAG amplifiers will be diode-pumped at a wavelength of $940 \mathrm{~nm}$. This is a prerequisite for achieving high repetition rates (light amplification duration 1 millisecond and repetition rate $10 \mathrm{~Hz}$ ). The efficiency of DPSSL is inversely proportional to the temperature, for this reason the slab amplifier have to be cooled at a temperature in the range of $100 \mathrm{~K}-170 \mathrm{~K}$ with a heat flux of $1 \mathrm{MW}^{*} \mathrm{~m}^{-2}$. This paper describes the thermo-mechanical analysis for the design of the amplification laser head, presents a preliminary proposal for the required cryogenic cooling system and finally outlines the gain of cryogenic operation for the efficiency of high pulsed laser.
\end{abstract}

\section{INTRODUCTION}

The efficiency of a laser is strongly connected with the thermo mechanical deformations of amplification media. A great part of the amplification energy is transformed in heat inside the laser media. This heat flux introduces mechanical deformation which is called "thermal lensing". This phenomenon is due to the non uniform heat deposition and non uniform cooling. This "thermal lensing" impacts the quality of the laser beam (power density and wave front). For these both reasons, lot of simulations and experimental analyses are engaged to improve the design of the amplifier and its cooling system. When a material is cooled down its thermal properties are changing, the heat conductivity increases and the heat capacity decreases. So an increase of the thermal diffusivity is observed for operation at low temperature $(<200 \mathrm{~K})$. As a consequence, the thermal gradient inside the amplification material can be reduced. In addition, experiments and simulations [1-4] have shown that laser materials have better amplifier performance when cooled. All these enhanced properties drive the development of high pulsed lasers at low temperatures. However, the cooling of amplifying sections at low temperatures (150-170 K) will require a more complex and more expensive infrastructure especially for the production of refrigeration capacity.

\section{MATERIAL AND DESIGN AMPLIFIERS}

\subsection{Amplification section design}

To avoid a very large thermal gradient in the crystal medium, the amplification medium has to be actively cooled. For this purpose, the outer surface of the ceramic is cooled by a forced convection with forced flow circulation. To increase the heat exchange surface, the amplifier is divided into several slabs separated by cooling channels as shown in figure 1 .

Helium gas has been chosen as a coolant gas for two major reasons. The first one is optical consideration [5]. The Helium refractive index is the lowest index for gases (10 times less than

This is an Open Access article distributed under the terms of the Creative Commons Attribution License 2.0, which permits unrestricted use, distribution, and reproduction in any medium, provided the original work is properly cited. 


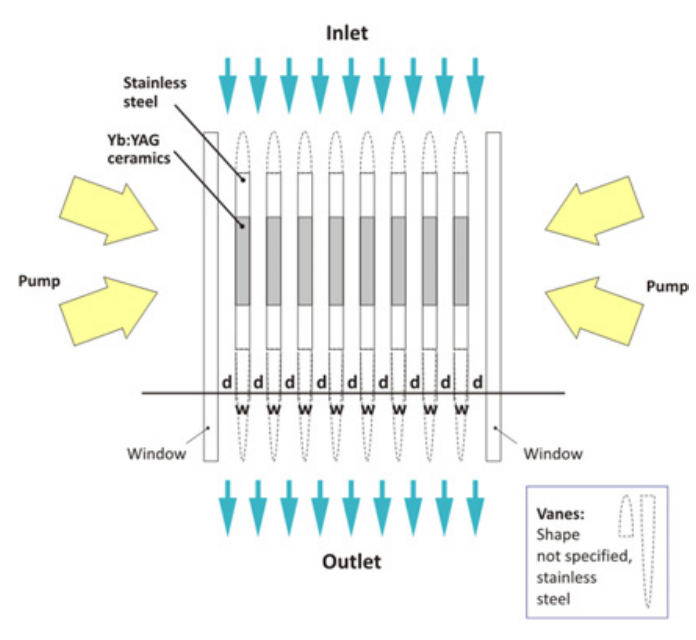

Figure 1. Schematic cross section of the cryogenic amplifier head.

Nitrogen). The second one is thermal consideration. Helium gas has a high heat capacity $(5.2 \mathrm{~J} / \mathrm{g} / \mathrm{K})$ and the temperature increase during a laser shot will be low. The combination of these two properties shows that helium gas is a good candidate as a cooling gas.

\subsection{Laser efficiency}

The properties observed at low temperature give lower dilatation coefficient and higher thermal diffusivity than at room temperature and would therefore facilitate the amplifier design [3].

Many experiments [1-3] show that the optical-to-optical efficiency of the amplification with YAG lasers doped $\mathrm{Yb}$ increases when the temperature of the amplification media is decreasing. This efficiency, which is $15 \%$ at room temperature, can reach $40 \%$ at a temperature in the range of $80-120 \mathrm{~K}$.

\section{THERMOMECHANICAL ANALYSIS}

The amplification medium is surrounded by a non active frame (undoped) which is here for fabrication and assembling reasons. In this case during the laser shots only the inner part is heated and compressive forces appear in the inner media. Crystals are very brittle and the stresses developed in the media have to stay lower than the stability stresses. This criterion is determined by coupling the cooling parameters and the mechanical properties of the crystals. The stability criterion minimizes the media deformation and "thermal lensing" effect. The following figure shows the scheme of the cooling.

The gas has a temperature $\mathrm{T}_{\infty}$ at the upstream and a heat exchange coefficient $\mathrm{h}$ at the interface surfaces. For uniform cooling all along the cooling channels, the temperature difference $\Delta \mathrm{T} 1$ in cooling gas is taken $<1 \mathrm{~K}$.

To remove the heat power generated in the crystals, a second $\Delta \mathrm{T} 2$ appears between the gas and the solid medium. Finally, a third $\Delta \mathrm{T} 3$ appears in the crystals along the crystal due to the helium flow. We suppose that $\Delta \mathrm{T} 2$ and $\Delta \mathrm{T} 3$ exist only in the active medium and the temperature of the surrounded non active medium stays at $\mathrm{T}_{\infty}$ as shown in the figure 2 . In this situation, compressive forces appear in the inner medium and buckling stresses could be developed. The compressive forces [6] depend on $\Delta \mathrm{T}$ inside the crystals. Compressive forces can introduce bucking phenomena if they are greater than a limit 


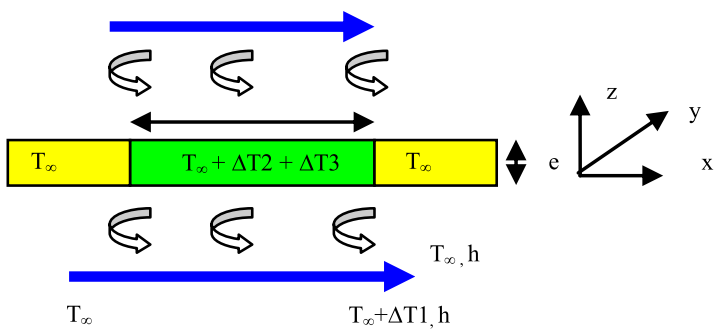

Figure 2. Scheme of an amplification plate for the thermo mechanical analysis.

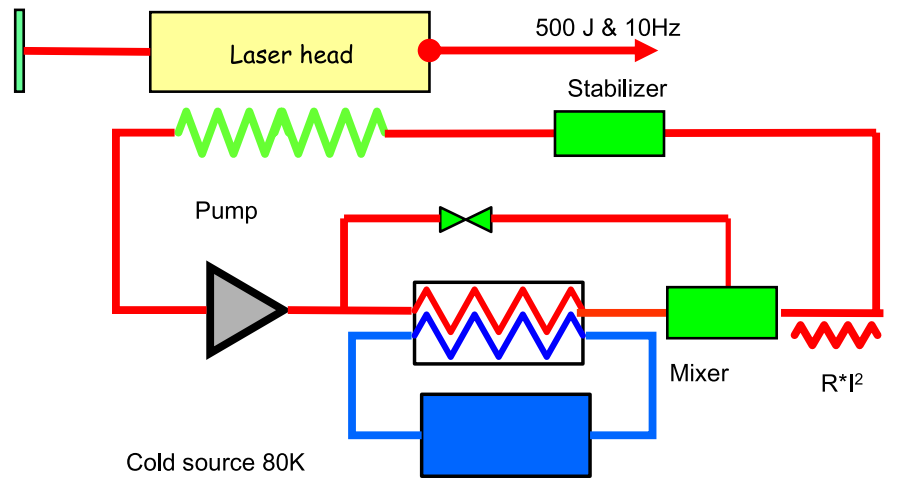

Figure 3. General scheme of the cooling loop.

value $\left(\sigma_{m}\right)$ called "stability value" [6] expressed in equation (1).

$$
\sigma_{m}=2 * \frac{\pi^{2}}{L_{1}^{2}} * \frac{E * e^{2}}{12 *\left(1-v^{2}\right)} .
$$

Where $E$ is the Young modulus, $v$ is the Poisson coefficient,

\section{COOLING SYSTEM}

\subsection{Scheme of the cooling loop}

Such lasers are pulsed laser and have a typical duty cycle with the duration of the pumping phase (light amplification) of 1 millisecond and a repetition rate of $10 \mathrm{~Hz}$. In these conditions the heat deposition will be considered as adiabatic, and for the calculation of the cooling system, an averaged power is considered.

A general scheme of the required cooling loop for high pulsed laser amplifiers is presented in figure 3 with a dedicated forced flow circulation in the amplifier cell. The main components of the loop are: the amplifier cell, a flow stabiliser, the temperature mixing chamber, a fine electrical heater, a circulating pump and a heat exchanger with a cold source. To reach the working temperature of the slab $(150-170 \mathrm{~K})$, a cold source at $80 \mathrm{~K}$ with a bypass is used. In this way the temperature of the slab can be adjusted in a large range $(90 \mathrm{~K}-170 \mathrm{~K})$ for laser performance studies. Additional heating by Joule effect can finely adjust the temperature. 


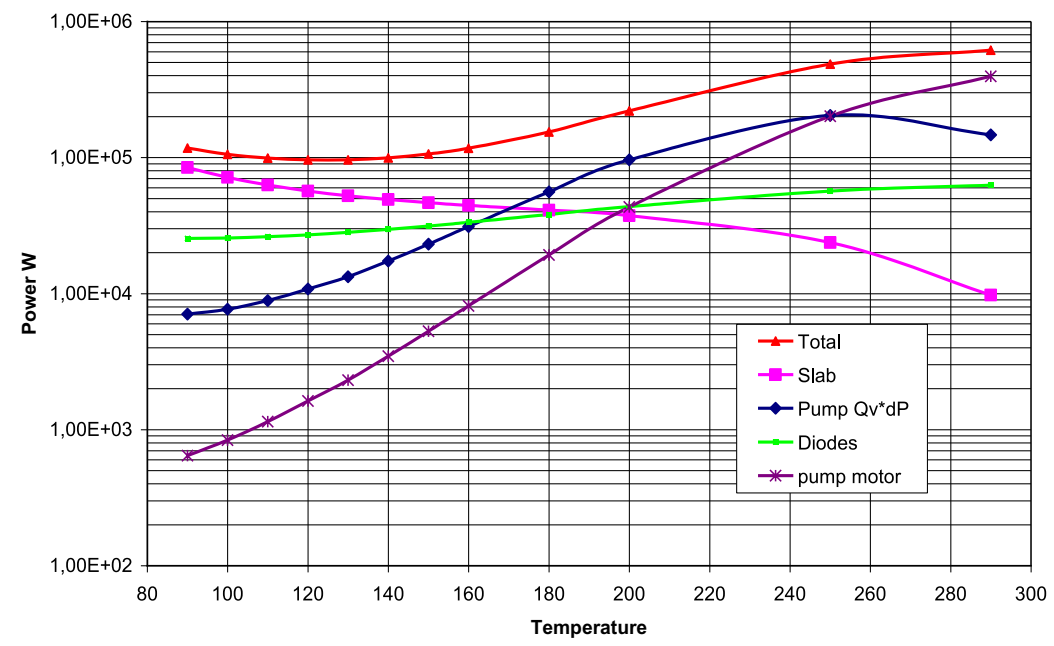

Figure 4. Equivalent electrical power required for a beam laser $(500 \mathrm{~J}$ at $10 \mathrm{~Hz})$

\subsection{Preliminary analysis for required energy}

According the operating temperatures, the total electrical power required to safely operate a high pulsed laser will depend of laser/diode performances and cooling efficiency. Figure 4 presents preliminary estimations of such total electrical power as a function of temperature.

Operating at low temperatures will reduce the total electrical consumption (ratio 1:3). One can note that for temperatures below $200 \mathrm{~K}$, the required energy is not very dependent on the temperature. This is explained by the fact that the decrease in Carnot efficiency for the cooling system is partly compensated by the increased efficiency of laser pumping for the lower temperatures.

\section{CONCLUSION}

The objective of operating DPSSL at low temperature is to improve the overall performance of these high pulsed lasers. This preliminary study shows that special attention including thermo mechanical analysis is required for the design of amplifiers operating at low temperature. The associated cryogenic system is standard but will require specific studies to fulfill the complex cooling requirements in the amplifier cell. Finally, preliminary estimations for operating a cryogenic DPSSL facility have shown that the total electrical consumption is drastically reduced when operating at low temperature.

\section{References}

[1] B. J. Le Garrec , G.L. Bourdet, E. Marmois, V. Cardinali, Solid state lasers and amplifiers, vol. 7721 (2010)

[2] B. J. Le Garrec, G.L. Bourdet, V. Cardinali, Fusion science and technology, vol. 56 (2009)

[3] D. C. Brown. IEEE journal of selected topics in quantum electronics, vol. 11, no. 3 (2005)

[4] A.J. Stone, A. Stejskal., Metrologia 41 (2004), page 189-197

[5] S. P. Timoshenko, J. M. Gere, Theory of stability, $2^{\text {nd }}$ edition, Mc Graw-Hill 